\title{
A multilevel model for movement rehabilitation in Traumatic Brain Injury (TBI) using Virtual Environments
}

\author{
Peter H. Wilson, Patrick Thomas, David Shum, Jonathan Duckworth, Mark Gugliemetti, Heiko \\ Rudolph, Nick Mumford, and Ross Eldridge
}

\begin{abstract}
This paper presents a conceptual model for movement rehabilitation of Traumatic Brain Injury (TBI) using virtual environments. This hybrid model integrates principles from ecological systems theory with recent advances in cognitive neuroscience, and supports a multilevel approach to both assessment and treatment. Performance outcomes at any stage of recovery are determined by the interplay of task, individual, and environmental/contextual factors. We argue that any system of rehabilitation should provide enough flexibility for task and context factors to be varied systematically, based on the current neuromotor and biomechanical capabilities of the performer or patient. Thus, in order to understand how treatment modalities are to be designed and implemented, there is a need to understand the function of brain systems that support learning at a given stage of recovery, and the inherent plasticity of the system. We know that virtual reality (VR) systems allow training environments to be presented in a highly automated, reliable, and scalable way. Presentation of these virtual environments (VEs) should permit movement analysis at three fundamental levels of behaviour: (i) neurocognitive bases of performance (we focus in particular on the development and use of internal models for action which support adaptive, on-line control); (ii) movement forms and patterns that describe the patients' movement signature at a given stage of recovery (i.e, kinetic and kinematic markers of movement proficiency), (iii) functional outcomes of the movement. Each level of analysis can also map quite seamlessly to different modes of treatment. At the neurocognitive level, for example, semi-immersive VEs can help retrain internal modeling processes by reinforcing the patients' sense of multimodal space (via augmented feedback), their position within it, and the ability to predict and control actions flexibly (via movement simulation and imagery training). More specifically, we derive four key therapeutic environment concepts (or Elements) presented using VR technologies: Embodiment (simulation and imagery), Spatial Sense (augmenting position sense), Procedural (automaticity and dual-task control), and Participatory (self-initiated action). The use of tangible
\end{abstract}

Manuscript received April 30, 2006. This work was supported in part by an Australian Research Council (ARC) Linkage Grant LP0562622, and Synapse Grant awarded by the Australia Council for the Arts.

P.H. Wilson is with the Division of Psychology, RMIT University, Melbourne, Australia. (Phone 613-9925-2906; fax: 613-9925-3587; email: peter.h.wilson@rmit.edu.au)

P. Thomas is with the School of Curriculum, Teaching and Learning, Griffith University, Brisbane, Australia (email: p.thomas@griffith.edu.au).

D. Shum is with the School of Psychology, Griffith University, Brisbane, Australia (email: d.shum@griffith.edu.au).

J. Duckworth is an artist with Metraform Inc.. He is also a $\mathrm{PhD}$ candidate with the School of Creative Media, RMIT University.

M. Gugliemetti is an artist with Metraform Inc.. He is also a Lecturer with the School of Art and Design, Monash University.

H. Rudolph is a Lecturer in the School of Computer and Electrical Engineering (SECE), RMIT University, Melbourne Australia.

N. Mumford is a PhD candidate with the Division of Psychology, RMIT University.

R. Eldridge is a PhD candidate with SECE, RMIT University. media/objects, force transduction, and vision-based tracking systems for the augmentation of gestures and physical presence will be discussed in this context.

\section{INTRODUCTION}

$\mathrm{T}$ RADITIONAL approaches to movement rehabilitation are steeped in the language of neuro-maturational theory. These approaches support methods that are designed to re-train movement (like gait) through a normal developmental sequence-e.g., [1]. Other techniques target sensory integrative processes using assisted movement (see Ref. [2]). By and large these approaches are not evidence-based, nor reflect the embodied nature of action. We propose an alternative approach that blends concepts from ecological systems theory with advances from the cognitive neuroscience of action (particularly the concept of internal modeling as a set of control processes). This hybrid approach does not seek an artificial separation between motor, cognitive, affective, and biological systems but views motor control and learning as determined by multiple and interactive constraintsindividual, task, and environmental. The approach is multilevel, acknowledging both bottom-up and top-down processes. We consider the neuromotor bases of movement control and learning, and the impact of cerebral insult on them, as well as higher-order control strategies that are used by the individual to negotiate novel tasks and to monitor their performance. We start with the patient's goal for action and consider how guided exploration can enhance the learning of functional skills. We also suggest that recovery will involve the motor system recalibrating the relationship between motor commands and their sensory consequences, the basis of feedforward control. Finally, we take into account the fact that systems of executive control, working memory, and motor intention are most susceptible to TBI, and rate-limiting factors in the recovery process.

Perhaps the most influential ideas drawn from ecological and dynamical models of movement are that motor control is an emergent property of the biological system, and that direct perception-action couplings support movement [3, 4]. The systems approach, thus, correctly views movement as an embodied phenomenon, where movement synergies and patterns are emergent phenomena that arise by the dynamic interplay of the biological system with its environment. The correspondence between the perceptual properties of different objects and events are mapped fairly directly to the action systems of the performer. Thus, the need for internal representations is downplayed under this scheme, whether they are for object, goal, decision or planning purposes. The 
performer does not need to construct object representations and the like in order to act, but rather "planning" is implicit in the perception-action cycle. The approach has a lot to commend itself by not drawing an artificial distinction between the performer and the natural constraints of his performance. For example, Gibson and others have talked about the importance of optic flow in specifying the structure of the 3-D world. This information can only be specified meaningfully as a consequence of self-motion [5]. The human system has developed these action systems to the extent that they have some adaptive significance for movement and interaction. In short, the legacy of evolution and selfmovement is the "direct perception" of affordances for action. What this suggests, then, is the existence of certain "motor primitives" that are common to all by virtue of our ancestral heritage; perceptual-motor systems that enable self-to-target and object-to-object relations to be specified are a prime example. However, identifying these so called invariant features of performance in neurobiological terms has been elusive.

The ecological view of Gibson and his contemporaries has been taken a step further to explain individual differences in movement control. It is argued that any movement, however complex, is specified by the biological constraints of the individual performer, and their interaction with task and environmental factors (see Figure 1). Shumway-Cook and Woollacott [6] do a nice job of modeling this 3-way interaction. Individual constraints can be divided into three (interactive) sub-components: the basic action systems themselves including biomechanical constraints, perceptual systems contributing to action, and cognitive processes (i.e., attention, memory, and motivation-emotion). Importantly, all three sub-components of the individual are generally compromised in TBI. At some basic level, these components interact to support motor control and learning capabilities. Task constraints can be classified according to the specific features of a task that regulate how a movement might unfold. While taxonomies vary, tasks are best categorized by function and intention from an ecological perspective: locomotion and stability, propulsion (of objects), reception, and orientation with respect to objects, terrain, or events [7]. Finally, environmental constraints concern both the regulatory features of the movement terrain and to-be-manipulated object(s), social supports, and background conditions like noise and lighting. In sum, these principles support a task-specific approach to re-training; minimal generalization is predicted as a result of re-training. Crucially, what ecological (or taskspecific) approaches neglect, however, is top-down control of action and the specification of universal neuromotor "constraints"; both factors suggest possibilities for positive transfer or movement generalization to the extent that metacognitive control and/or neuromotor primitives can be treated in some way.

To round out our model of motor behaviour, we introduce the notion of neurocognitive bases, drawing on neuroscience. These are basic motor control processes whose function constrains movement proficiency across tasks and time. For intact systems, we refer to (emerging) brain systems that support movement over the course of development; e.g., differentiation of dorsal stream networks responsible for coding movement in body-centred coordinates [8]. What follows then are three levels of movement behaviour and analysis: (i) neuromotor bases of performance (we focus in particular on the development and use of internal models for action which support adaptive, on-line control); (ii) movement forms and patterns that describe the patients' movement "signature" at a given stage of recovery (i.e, kinetic and kinematic markers of movement proficiency), (iii) functional outcomes of the movement. We focus more on the first level in this paper as its represents a significant advance in thinking about movement dysfunction in TBI and methods of rehabilitation, facilitated by use of VEs.

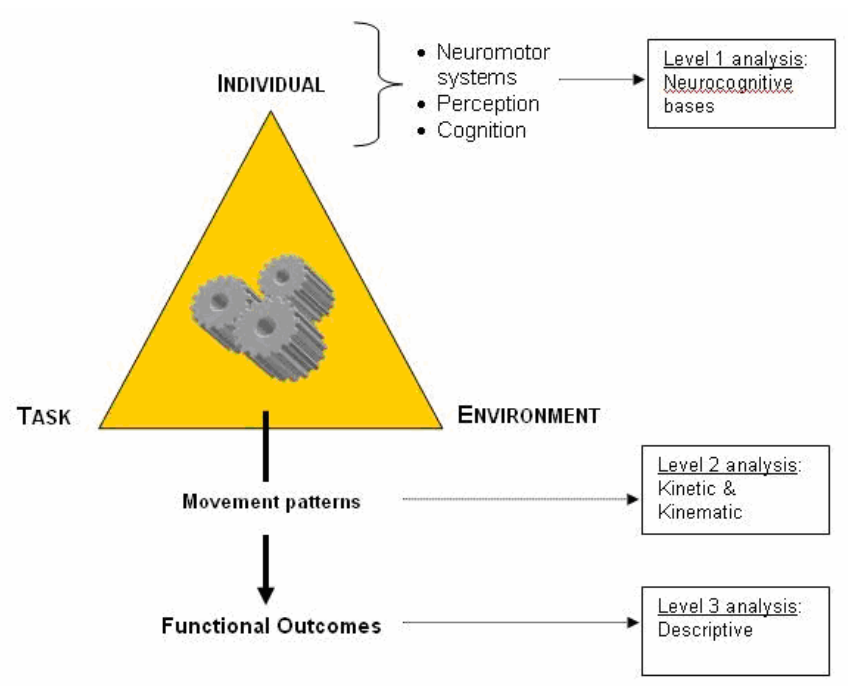

Figure 1: A simplified hybrid model of movement control and analysis (adapted from Ref. [6]).

\section{LEVELS OF MOVEMENT BEHAVIOUR AND ANALYSIS}

\section{Level 1 Analysis - Neuromotor bases of action}

Two crucial issues limit the effectiveness of current movement rehabilitation practices: (i) the extent to which clients can re-calibrate internal models for action (inverse and feedforward models), and (ii) how readily movement skills learned in a rehab setting can generalise to the real world, the limiting factor being the development of procedural control (or automaticity). Internal modeling has become an important concept in neurocognitive models of motor function $[9,10]$. Internal models are of two types: So called forward models use a copy of the motor command (or efference copy) to predict the future state of the moving $\operatorname{limb}(\mathrm{s})$. By comparison, the inverse model (or Controller) generates the motor commands necessary to achieve a task goal state. For example, neurons of the intraparietal sulcus (IPS) have a crucial role in coding target position in body-centred coordinates [11]. 
Forward models contribute to volitional control by anticipating and cancelling out the sensory consequences of a given movement enabling the mobile observer to distinguish between self-produced and externally-induced motion. The original motor intention appears to emanate from premotor cortex, however, with it, the parietal lobe forms part of a reciprocal cortico-cortical sensorimotor loop that appears to subserve the forward planning process [12]. Internal models generated by the parietal lobe would, thus, play a role in monitoring intentions and motor plans at high levels of representation, verifying whether actions match their intended goals. As well, by anticipating the sensory outcome of a movement before it is executed, forward models can be engaged in imagery training to select optimal action sequences $[10,13]$. In short, the ability to generate an accurate internal model of how an intended movement will unfold (i.e., feedforward modeling) is crucial to adaptive control and recovery of function. Unfortunately, this process is commonly impaired in TBI and hemiparesis [14], but neglected in intervention [15].

In the area of developmental motor disorders, our group has developed a neurocognitive model of motor dysfunction that highlights the importance of internal modeling to the acquisition of functional skills $[16,17]$. This model has clear implications for movement rehab. For example, based on this framework, we have developed motor imagery training protocols for adapted movement education using an interactive CD-Rom [18]. Here digitized models and verbal scripts are used to prime the performer about effective movement form and outcome. This modeling and cuing process is thought to encourage the performer to more easily simulate anticipated outcomes for movement, enhancing feedforward control [16]. Supporting this argument are findings that show performance benefits across skill domains.

With respect to the development of automaticity, basic movement skills are often learned in very controlled environments. Once the patient is required to apply skills in real-world settings, demands on attention and working memory often exceed their processing and response capabilities [19]. As such, performance declines markedly, along with their motivation to achieve and movement confidence. There are few systematic approaches to rehabilitation that permit stimulus characteristics to be varied in a graded fashion, recorded with precision, and that fit the patient's changing response capabilities. In short, rehabilitation techniques are needed to shape the ability to plan and execute movements in complex workspaces.

Our group has a strong interest in accelerating rehab, moving the client's performance from a controlled, slow, effortful mode of processing to an automatic, fast, and timeefficient mode. Part of the transition is supported by gains in feedforward control, but also by development of cognitive techniques that enhance memory skill and executive control. To this end, we have developed process-oriented treatment programs for children with coordination problems [18] and functional skills for patients with TBI [20]. For example, by supporting goal-directed action with external memory prompts, Shum and colleagues have demonstrated improved functional performance on a range of everyday tasks, including those performed over remote time frames [20, 21, 22]. Their results also suggest that for actions performed in real time, under high information load, cuing can assist patients to re-learn motor routines.

\section{Level 2 - Movement Form and Strategy}

Movement form and efficiency reflect the particular movement solutions adopted by the recovering patient, which may or may not realize the intended functional goal. Indeed, successful completion of functional skills may be impossible or very laboured during the early stages of recovery. Kinematic and kinetic parameters can help the clinician describe what aspects of coordination, timing, synergy, or force production might not be adaptive [23]. One point of tension from the ecological perspective is that while it supports an idiographic approach to movement behaviour, there is enough commonality in the biomechanics of bipedal motion that basic movement typologies can be identified. For example, in the case of normal development, researchers have identified control parameters that explain shifts between different modes of coordination in the mobile performer. For example, the transition from crawling to standing is constrained by muscle strength, among other factors [24]. Other work has described changes in movement kinematics that occur with maturation, particularly those associated with the development of walking and reach-to-grasp movements (e.g., Ref. [25]). There is sufficient evidence to show that the recovering patient with TBI also undergoes transitions in movement form, in many cases reflecting changes in control parameters like strength, tone, and flexibility [23]. Therapeutically, Holden and Dyar [26] have applied this principle to good effect by clever use of virtual modeling, and reproduction, encouraging new synergies and more efficient end-point control.

\section{Level 3 - Functional performance}

Assessment of functional outcomes is a standard focus for rehab therapists. Importantly, the assessment (or therapeutic) task needs to hold clear motivational significance for the patient and ideally the action needs to be purposive; it is, after all, at the level of goal representation that movement intention is mapped to planned trajectories/paths of motion. The use of functionally significant target objects will support the process of internal modeling. In the intact system, inverse models are generated that specify target locations in body-centred coordinates, as well as the force-timing parameters that are needed to move the effector toward a virtual endpoint [10]. Notably, goal-directed actions to significant objects have been shown to produce smoother and faster reaching movements than non-object conditions for paretic patients [27].

In the case of reaching in TBI, performance is related to a number of biomechanical constraints including increased muscle tone as a result of spasticity, reduced agonist strength, and reduced coupling between elbow and shoulder. Crucially, 
repeated practice of functional skills is needed to improve muscle strength and restore these couplings [28]. One of the major impediments to recovery, then, is the patient's ability to engage in the therapeutic regime and to persist. Again, we come back to the importance of presenting tasks and task environments in a meaningful and stimulating way. In the final section we discuss how this can be achieved using virtual environments. As well, these environments enable presentation of graded challenges. Therapeutically, this will help shape functional proficiencies, ensuring that gains made in the clinical environments can be transferred more seamlessly to the real world.

\section{NEUROCOGNITIVE BASES OF PERFORMANCE IN TBI}

TBI can impact on physical performance, cognition, and emotional regulation. Depending on the type of insult, impairments in movement can severely limit the patient's ability to engage in activities of daily living, including basic mobility [29]. Enhancing rehabilitative processes in the early stages following cerebral insult is one of the great challenges for therapists; it is during the first 6 months post-TBI that damaged neural systems are most receptive to stimulation and re-organization [15]. Movement imagery, multimodal/augmented feedback, and procedural (or dual-task) control represent three modes of intervention at a neurocognitive level that hold great promise for remediation of TBI. Our working model has some important things to say about how these modalities might be implemented, and their presentation supported by virtual environments.

Movement imagery. There is strong evidence that motor imagery training in unimpaired populations can affect substantial gains in skill performance, even in the absence of any change in physical practice [30]. Motor imagery protocols have also recently been shown to benefit TBI patients. For example, we know that TBI and hemiplegic patients retain the ability to mentally simulate movements that they can no longer perform post trauma [31]. Indeed, imagery training has been used to promote recovery from TBI, mild hemiplegia, and stroke as it provides a means of stimulating brain circuits compromised by trauma and/or hemorrhage. Notably, recent work shows that by adopting a first-person (or egocentric) perspective when mentally simulating an action, cortical motor networks are activated most strongly, promoting efficient motor timing [32] and refining forward models for action. We have used this principle successfully in children with developmental motor disorders by shaping the internal simulation of movement [18]. We discuss its application to TBI in the following section.

Multimodal representation. We also know that experience-dependent synaptic wiring and replacement shares many of the same processes that drive normal motor learning [15]. To maximise the opportunity for synaptic re-growth and re-tuning, the damaged motor system must receive varied but correlated forms of sensory input during the early phase of recovery; this input encourages development of a sense of multimodal space and of the spatial relationship between performer and objects [33, 34]. For example, simple forms of augmented feedback (e.g., visual analogue displays of limb position from different viewpoints) have been shown to support the re-training of basic movement patterns in TBI [19]. In the following section we discuss how this application can be extended by designing movement-tracked exercises (in an interactive VE) that augment and amplify user actions, reinforcing the patient's (multimodal) sense of position in space.

Procedural control. Skill execution in the early stages of rehabilitation is constrained by disruptions to attentional and working memory systems [35]. TBI patients will initially find it difficult to attend to both a primary task (e.g., walking) and a simultaneous secondary task (e.g., signal detection). The goal of rehab is to support the development of procedural knowledge (or automaticity) as skills are relearned. This reduces the processing load associated with performance, enabling the patient to allocate attention to other events and contingencies in the environment. In the dual-task paradigm, the extent to which effective performance on both tasks is achieved concurrently will reflect the level of automaticity in motor skill execution; in turn, this has implications for functionality. This type of paradigm is currently lacking in rehabilitation, mainly due to limitations in workspace design. One important goal of therapy, therefore, is to train patients to be more flexible in controlling more than one cognitive operation at one time while moving.

\section{IMPLICATIONS FOR THE DESIGN OF VES}

The use of VR technology to facilitate recovery from TBI is in the early stages of development, but has been shown to assist patients to regain basic mobility skills and balance [36], and manual dexterity [37]. The technology is valuable because it permits flexible presentation of learning workspaces and of augmented (multimodal) feedback. In the case of hand function, for example, real time 3-D visual scenes can be displayed to prompt perceived possibilities for interaction. Digitally enhanced visual and auditory feedback combined with a compelling sense of realism can be conveyed to patients while they perform simple movements. Presented within the context of a game, augmented feedback has been used to improve range of motion, hand strength, and movement speed [37].

The generic advantages of VR technology over traditional methods have been well documented by Ref. [38]: presentation of ecologically valid testing and training scenarios; flexible control of stimulus conditions to enhance learning; safe presentation of assessment and training situations; enhanced generalization of learning; standardization of assessment and training protocols which supports evaluation of training components; enhanced user participation, and ability to complement conventional therapies. VR technology can provide a highly structured and replicable (virtual) environment for TBI patients under which basic response capabilities can be assessed, as well as an 
enhanced degree of interactivity between the patient and learning task.

As mentioned, approaches to movement rehab in TBI have traditionally been designed to re-establish basic mobility patterns and manual skills using (hands-on) assisted therapy. These approaches are labour and cost intensive; they require one-to-one physical and occupational therapy over an extended period using a variety of props, in relatively large workspaces [39]. Accordingly, the capacity of rehab services to cater to multiple clients with differing needs is severely limited. Unfortunately, this reality does not accord with evidence that intensive intervention is required in the early stages post-TBI in order to optimize recovery [2]. The development of technologies that can help rehab providers accelerate the recovery process is a core issue in advancing rehab practices for TBI.

The explanatory model we have proposed has clear implications for the development of (therapeutic) environment concepts (or Elements) that promote re-calibration of motor control processes and re-acquisition of functional skill in TBI. The overarching goals are to: draw patients into these environments in a graded way and to invite interaction; foster a clear goal representation at each task level; provide external prompts and feedback that encourage a clear sense of control and position in space, and to encourage patient's to explore their movement capabilities in a self-directed fashion. We identify four key Elements to assessment and training: Embodiment (simulation and imagery), Spatial Sense (augmenting position sense), Procedural (automaticity and dual-task control), and Participatory (self-initiated action).

Both the Embodiment and Spatial elements are design concepts that serve the internal modeling process. For Embodiment, we suggest use of different methods of motor imagery training to enhance the patients' sense of body schema and predictive control. For example, mental rotation exercises involving limb-related stimuli can be presented using a shaping procedure; behavioural studies show that responses to these stimuli conform to the same biomechanical constraints as real movements [34] and enlist the same motor planning networks [40]. VE-based simulations of translational and rotational limb movement, together with verbal prompting might be used to encourage the patient to adopt an internal (or first-person) perspective when making "judgements" on mental rotation tasks, thereby stimulating (cortical) motor planning networks. These external supports can then be removed over time. The integrity of internal modeling can also be measured by the pattern of response on the mental (limb) rotation tasks-i.e., regression estimates defining the relationship between response time and angle of rotation [17].

For the Spatial Sense element, orientation in space can be enhanced by presenting feedback that reinforces changes in the flow of visual, auditory, and tactile information that occur with self-movement. Position in space is intimately tied to the ability to anticipate spatiotemporal flow. Augmented feedback can help the patient form a dynamic representation of their position in space, and assist judgments about time-to-contact relative to objects and the (virtual) terrain [15]. In this way, internal modeling processes are supported and re-calibrated. An enhanced sense of physical presence can also assist clients to re-establish prospective judgments and movement plans over larger time scales (Roche, Fleming \& Shum, in press). Augmented feedback about the effects of self-movement can be presented using different sensory modalities including vision (visual field displacement), sound (calibrated to the timing of movement sequences), and tactility (real-time dynamic changes in the VE that respond to physical movement or changes in force production). These multimodal supports do not feature in current rehab practice.

For Procedural control, a semi-immersive dual-task paradigm can support both assessment and treatment. For both mobility and reach-to-grasp skills, the VEs need to vary task constraints in a graded and systematic way. For example, task load can be manipulated when simulating walking through a virtual terrain by varying the type and number of obstacles. During the acquisition phase of skill learning, the function of attention and memory can be supported by visual cuing, and then, gradually, removed; e.g., visual cues and verbal prompts can signal attention to obstacles and prospective events. These cues can be scaled to the particular client's stage of learning, and are highly replicable. This will shape procedural learning, enhancing dual-task control (e.g., processing other invitations to action while already in motion). Dual-task costs can be measured at different stages of recovery, providing an index of automaticity for fundamental movement tasks.

The Participatory element concerns methods to re-engage self-directed movement and exploration by encouraging the patient to exert direct control over the stimulus environment. It is designed to build intent and purpose in the action predispositions of patients with TBI, qualities often lacking during the acute phase of recovery. The stimulus objects may be floor or wall projected, concrete or abstract in design, but dynamic in their response to the direction of the performer. For example, using very simple movements, patients could explore their ability to control objects to assemble a visual and/or aural event. This will provide an immediate form of reinforcement for action, building motivation to engage in therapy.

\section{CONCLUSION}

A blending of constraint-based ecological systems theory and cognitive neuroscience can provide a viable model of movement control in TBI, one that supports a multilevel approach to assessment and rehabilitation. We show that at the neurocognitive level, internal modeling, multimodal integration, and procedural learning are all important control concepts that help explain some of the fundamental performance limitations of patients with TBI. These concepts can be treated using a brain systems approach to therapy. VR technologies generally, and VEs more specifically, can provide the ideal platform for this approach. We also envisage that the program of virtual environments can be easily adapted to find a broader application in the treatment of movement 
dysfunction in other neuropsychological disorders (e.g., stroke and ischemic disorders), and movement disorders associated with aging (e.g., Parkinson's Disease and Huntington's chorea).

\section{REFERENCES}

[1] B. Bobath, Adult hemiplegia: Evaluation and treatment. London: Heinemann Medical Books, 1989.

[2] S. Blanton, L. Porter, D. Smith D, and S.L. Wolf, "Strategies to enhance mobility in traumatic brain injured patients," in Rehabilitation of the adult and child with TBI, $3^{\text {rd }}$ ed., M. Rosenthal et al. (Eds.). Philadelphia, PA: F.A. Davies, 1999, pp. 219-241.

[3] J.J. Gibson, The senses considered as perceptual systems. Boston: Houghton Mifflin, 1966.

[4] M.T. Turvey, "Preliminaries to a theory of action," in Perceiving, Acting and Knowing: Toward and Ecological Psychology, R. Shaw and J. Bransford (Eds.). Hillsdale, NJ: Erlbaum, 1980.

[5] W.H. Warren, "Self-motion: Visual perception and visual content," in Handbook of perception and cognition, vol. 5, W. Epstein and S. Rogers (Eds.). New York: Academic Press, 1995.

[6] A. Shumway-Cook and M. Woollacott, Motor control: Theory and practical applications, $2^{\text {nd }}$ ed. Baltimore, ML: Lippincott, Williams, and Wilkins, 2001.

[7] W. Davis and A.W. Burton, "Ecological task analysis: Translating movement behavior theory into practice," Adapted Physical Activity Quarterly, vol. 91, pp. 154-177, 1991.

[8] B.J. Casey, N. Tottenham, C. Liston, and S. Durston, "Imaging the developing brain: what have we learned about cognitive development?," Trends in Cognitive Science, vol. 9, pp. 104-110, 2005.

[9] C.D. Frith, S.J. Blakemore, and D.M. Wolpert, "Abnormalities in the awareness and control of action," Phil Trans Royal Soc London, vol. 355, pp. 1771-1788, 2000.

[10] D. Wolpert, "Computational approaches to motor control," Trends Cog Sci, vol. 1, pp. 209-216, 1997.

[11] T. Flash and T.J. Sejnowski, "Computationl approaches to motor control," Current Opinion Neurobiol, vol 11, pp. 655-662, 2001.

[12] A. Sirigu, E. Daprati, S. Ciancia, P. Giraux, N. Nighoghossian, A. Posada, and P. Haggard, "Altered awareness of voluntary action after damage to the parietal cortex," Nature Neurosci, vol. 7, pp. 80-84, 2004.

[13] S. Katschmarsky, S. Cairney, P. Maruff, P.H. Wilson, P. Tyler, and J. Currie, "The ability to execute saccades on the basis of efference copy: Impairments in children with developmental coordination disorder," Experimental Brain Research, vol. 136, pp. 73-78, 2001.

[14] R.F. Beer, J.P.A. Dewald, and W.Z. Rymer, "Deficits in the coordination of multijoint arm movements in patients with hemiparesis; Evidence for disturbed control of limb dynamics," Experimental Brain Research, vol. 131, pp. 305-319, 2000.

[15] F. Morganti, A. Gaggioli, G. Castelnuovo, D. Bulla, M. Vettorello, and G. Riva, "The use of technology-supported mental imagery in neurological rehabilitation: A research protocol," Cyberpsychol Behav., vol 6(4), pp. 421-427, 2003.

[16] P.H. Wilson, "Approaches to Assessment and Treatment of Children with DCD: An Evaluative Review," Journal of Child Psychology and Psychiatry, vol. 46, pp. 806-823, 2005.

[17] P.H. Wilson, P. Maruff, M. Butson, J. Williams, J. Lum, and P. Thomas, "Impairments In The Internal Representation Of Movement In Children With Developmental Coordination Disorder (DCD): Evidence From A Mental Rotation Task," Dev Med Child Neurol, vol. 46, pp. 754-759, 2004.

[18] P.H. Wilson, P. Thomas, and P. Maruff, "Motor imagery training ameliorates motor clumsiness in children," Child Neurol, vol. 17, pp. 491-498, 2002.

[19] M.T. Schultheis and A.A. Rizzo, "The applications of virtual reality technology for rehabilitation," Rehab Psychol, 46, pp. 296-311, 2001.
[20] D. Shum, D. Harris, and L. O'Gorman, "Effects of severe traumatic brain injury on visual memory," Journal of Clinical and Experimental Neuropsychology, vol. 22, pp. 25-39, 2000.

[21] E. Kendall, D. Shum, B. Lack, S. Bull, and C. Fee, "Coping following traumatic brain injury: The need for contextually sensitive assessment," Brain Impairment, vol. 2, pp. 1-9, 2001.

[22] A. Maujean, D. Shum, and R. MacQueen, "Effect of cognitive demand on prospective memory in individuals with traumatic brain injury," Brain Impairment, vol. 4, pp. 135-145, 2003.

[23] P.H. McCrea, J.J. Eng, and A.J. Hodgson, "Biomechanics of reaching: Clinical implications for individuals with acquired brain injury," Disability and Rehabilitation, vol. 24, pp. 534-541, 2002.

[24] E. Thelen, "Motor development as foundation and future of developmental psychology," International Journal of Behavioral Development, vol. 24, pp. 385-397, 2000.

[25] S. Schneiberg, H. Sveistrup, B. McFadyen, P. McKinley, and M.F. Levin, "The development of coordination for reach-to-grasp movements in children," Experimental Brain Research, vol 146, pp. 142-154, 2002.

[26] M.K. Holden and T. Dyar, "Virtual environment training: A new tool for rehabilitation," Neurology Report, vol. 26, pp. 62-72, 2002.

[27] C. Wu, M. Wong, K. Lin, and H. Chen, "Effects of task goal and personal preference on seated reaching kinematics after stroke," Stroke, vol. 32, pp. 70-76, 2001.

[28] M.F. Levin, "Interjoint coordination during pointing movements is disrupted in spastic hemiparesis," Brain, vol. 119, pp. 281-293, 1996.

[29] D. Shum, M. Valentine, and T. Cutmore, "Performance of traumatic brain-injured individuals on time-, event-, and activity-based prospective memory tasks," J Clin Exp Neuropsychol, vol 21, pp. 49-58, 1999.

[30] P.R. Thomas and G.J. Fogarty, "Psychological skills training in golf: The role of individual differences in cognitive preferences," Sport Psychologist, vol. 11, pp. 86-106, 1997.

[31] S. H. Johnson-Frey, "Stimulation through simulation? Motor imagery and functional reorganization in hemiplegic stroke patients," Brain and Cognition, vol. 55, pp. 328-331, 2004.

[32] T. Wolbers, C. Weiller, \& C. Borchel "Contralateral coding of imagined body parts in the superior parietal lobe," Cerebral Cortex, vol. 13, pp. 392-399, 2003.

[33] R.A. Anderson, L.H. Snyder, D.C. Bradley and J. Xing, "Multimodal representation of space in the posterior parietal cortex and its use in planning movements," Annual Review of Neuroscience, vol. 20, pp. 303330, 1997.

[34] P.H. Wilson, "Visual-motor, visual-perceptual, kinaesthetic, and visuoconstructional disorders," Developmental Motor Disorders: A neuropsychological perspective, Dewey and Tupper (Eds.). Guilford, 2004.

[35] D. Shum, K. McFarland, and J. Bain, "Effects of closed head injury on attentional processes: Generality of the Additive Factor Method," J Clin Exp Neuropsychol, vol. 16, pp. 547-555, 1994.

[36] H. Sveistrup, J. McComas, M. Thornton, S. Marshall, H. Finestone, A. McCormick, K. Babulic, and A. Mayhew, "Experimental studies of virtual-reality-delivered compared to conventional exercise programs for rehabilitation," Cyberpsychol Behav., vol. 6, pp. 245-249, 2003.

[37] A.S. Merians, D. Jack, R. Boian, M. Tremaine, G.C. Burdea, S.V. Adamovich, M. Recce, and H. Poizner, "Virtual reality-augmented rehabilitation for patients following stroke," Phys Ther, vol. 82, pp. 898$915,2002$.

[38] M.T. Schultheis, J. Himelstein, and A.A. Rizzo, "Virtual reality and neuropsychology," J Head Trauma Rehabil., vol 17, pp. 378-394, 2002.

[39] A.A. Rizzo, J.C. Buckwalter, and C. van der Zaag, "Virtual environment applications in clinical neuropsychology," in Handbook of virtual environments, K.M. Stanney (Ed.). Mahwah, NJ: Erlbaum, 2002, pp. 1027-1064.

[40] E. Mellet, L. Petit, B. Mazoyer, M. Denis, and N. Tzourio, "Reopening the mental imagery debate," Neuroimage, vol. 8, pp. 129-139, 1998. 